\title{
Grouping puts figure-ground assignment in context by constraining propagation of edge assignment
}

\author{
JoSEPH L. BROOKS AND JON DRIVER \\ University College London, London, England
}

\begin{abstract}
Figure-ground organization involves the assignment of edges to a figural shape on one or the other side of each dividing edge. Established visual cues for edge assignment primarily concern relatively local rather than contextual factors. In the present article, we show that an assignment for a locally unbiased edge can be affected by an assignment of a remote contextual edge that has its own locally biased assignment. We find that such propagation of edge assignment from the biased remote context occurs only when the biased and unbiased edges are grouped. This new principle, whereby grouping constrains the propagation of figural edge assignment, emerges from both subjective reports and an objective short-term edge-matching task. It generalizes from moving displays involving grouping by common fate and collinearity, to static displays with grouping by similarity of edge-contrast polarity, or apparent occlusion. Our results identify a new contextual influence on edge assignment. They also identify a new mechanistic relation between grouping and figure-ground processes, whereby grouping between remote elements can constrain the propagation of edge assignment between those elements. Supplemental materials for this article may be downloaded from http://app.psychonomic-journals.org/content/supplemental.
\end{abstract}

Edge assignment is a key aspect of figure-ground organization. When a dividing edge is assigned to one of its adjacent regions, that region becomes "figural" and is perceived as shaped along the edge and in front, whereas the other adjacent "ground" region is not shaped and appears behind the figural region (Baylis \& Cale, 2001; Driver \& Baylis, 1996; Rubin, 1921). This is illustrated by the change in perceived shape associated with reversals of figural edge assignment, as for the well-known faces-vase example. Furthermore, figural edge assignment affects the way that visual input is processed by brain regions that are involved in shape representation and object recognition (see, e.g., Appelbaum, Wade, Vildavski, Pettet, \& Norcia, 2006; Baylis \& Driver, 2001; Kourtzi \& Kanwisher, 2001).

Established image cues for determining figural edge assignment typically involve relatively local edge or region properties, such as convexity (Kanizsa \& Gerbino, 1976; Metzger, 1953; Stevens \& Brookes, 1988), symmetry (Kanizsa \& Gerbino, 1976; Rubin, 1921), relative size and contrast (Palmer, 1999; Rubin, 1921), relative position in the scene (Hulleman \& Humphreys, 2004; Vecera, Vogel, \& Woodman, 2002), or local edge-region grouping relationships (Palmer \& Brooks, 2008), among other image-based factors. Higher level factors such as attention (Baylis \& Driver, 1995; Driver \& Baylis, 1996; Vecera, Flevaris, \& Filapek, 2004; although see Huang \& Pashler, 2009) and previous experience (Peterson, 1994; Peterson \& Gibson, 1994a, 1994b) have also been suggested to play some role in determining figural edge assignment.
However, the possible influences of distant, contextual parts of an image on figural edge assignment elsewhere in that image remain less explored. Recently, Peterson and Salvagio (2008) demonstrated that the effectiveness of convexity for local figural edge assignment can be affected by more global context. For a bipartite display (i.e., comprising two adjacent regions) with a single critical dividing edge, the more convex region was only weakly biased toward figural status. However, when additional surrounding edges and regions were added together to form a chain of convex/concave/convex regions, the convex regions became significantly more likely to be chosen as figural. Evidently, the presence of the convexity cue at multiple edges affected the impact of that cue. Interestingly, this effect of context occurred only when the concave regions that intervened between the successive convex regions were similarly colored, thereby providing a homogeneous background made up of all the commonly colored concave regions. These results indicate that, under certain conditions, the strength of existing figural biases within a scene may be enhanced contextually by other edges/regions in the scene. However, we know of no examples to date in which figural edge assignment for a locally unbiased edge (i.e., in which no local figural cues are present) has been shown unequivocally to be determined by figural assignment of remote contextual edges elsewhere in the image (whose figural assignment is determined by their own local biasing factors that do not apply for the unbiased edge elsewhere). Thus, although Peterson

J. L. Brooks, joseph.brooks@ucl.ac.uk 
and Salvagio provided an initial contextual demonstration that a weak local cue can have more impact when combined with matching context, it still remains unknown whether figural assignment for a locally unbiased edge can be determined by propagation of figural assignment from a remote but locally biased contextual edge.

In the present article, we demonstrate that figural edge assignment of a locally ambiguous edge (i.e., with no local cues to figural assignment along that edge, nor in its adjoining regions) can, in fact, be determined by an assignment of remote contextual edges. We also investigate the boundary conditions for contextual edges to propagate their influence to other edges in this way. We propose that perceptual grouping can constrain whether or not a given contextual edge will influence figural edge assignment of the locally ambiguous edge. Specifically, when an unbiased edge is grouped with a separate, locally biased edge, we predict that the figural assignment of the latter will propagate to the former. But, we expect such propagation to be curtailed when biased and unbiased edges are not grouped together. This prediction arises intuitively from an ecological rationale. When two edges are grouped together, this indicates that those two edges are likely to belong to the same external source and hence may also be likely to have congruent figure-ground (depth) relations in the physical world, along the grouped edges. By contrast, for edges that are not grouped together by the visual system, corresponding figure-ground assignment may be less likely.

From a more formal perspective, the new prediction that we tested for the first time in the present study also appears to be in line with some recent computational models of edge assignment that, in practice, rely on "grouping" operations in order to propagate depth information from local T-junction cues to other locations along a complete or partially complete contour (Craft, Schutze, Niebur, \& von der Heydt, 2007; Heitger, von der Heydt, Peterhans, Rosenthaler, \& Kubler, 1998; Peterhans \& Heitger, 2001; Thielscher \& Neumann, 2008). In the present study, we tested for human vision, whether grouping operations do indeed serve as a mechanism for constraining the propagation of figure-ground assignment from one contour to another in separate parts of the image.

Our proposal that grouping should constrain propagation of edge assignment in this specific way appears to contrast with some other influential proposals about the relationship between figural edge assignment and grouping. For instance, Palmer and Rock (1994b) initially proposed that figure-ground assignment invariably arises before perceptual grouping, so that the latter should presumably not influence the former (although they later finessed this to acknowledge a possible role for interactive activation between successive stages of visual processing; see Palmer \& Rock [1994a, 1994b]). Their initial claim was largely based on an argument from first principles, which had assumed that figure-ground assignment must always operate first, to provide candidate items to undergo grouping. Our experiments directly tested whether instead grouping can, in fact, constrain figure-ground assignment (indicating the reverse influence) to determine whether or not figure-ground as- signment will be propagated from one contour to another. Although "figure-ground organization" (i.e., edge assignment) and "grouping" (i.e., perceptual linking of separate elements in an image) are often jointly discussed in many textbook chapters on Gestalt psychology, typically little or no explicit mechanistic relation is specified between the two types of process in such chapters, unlike in the specific proposal we tested in the present study.

In order to assess our proposal that figure-ground organization should propagate from contextual contours to unbiased contours, specifically only when the latter are grouped with the former, we created novel displays comprising two main "sections," plus an occluder separating them (Figure 1A). The top and bottom bipartite sections each comprised two differently colored regions (shown as black and white in Figure 1A), separated by a vertical contrast edge. The vertical edge for the locally biased section (bottom section in Figure 1A) was assigned by local figure-ground cues toward one or the other side (see below for a description of determining local cues, which involved moving dots in our initial experiments, but see the later experiments for different cues in static displays). The other unbiased-section (top section in Figure 1A) had no such local edge-assignment cues. A rectangular occluder (shown as gray in Figure 1A, actually red in our initial experiments) clearly separated the biased and unbiased sections in the displayed image. The original rationale for this occluder was to demarcate a clear separation in the image between biased and unbiased sections, but we return to its possible perceptual role later.

We measured whether edge assignment for the central vertical edge in the biased section of the display would affect edge assignment for the central vertical edge in the unbiased section, to produce a remote contextual effect. To test whether grouping would constrain any such propagation of edge assignment from biased to unbiased sections, we manipulated perceptual grouping between the central dividing edges (henceforth termed edge grouping) of the biased and unbiased sections. In Experiment 1 , the central vertical edges within biased and unbiased sections moved from left to right in an oscillatory manner (see the animations in the supplemental materials). These edges clearly grouped when they moved collinearly together with common fate (Figures 1B-1C). In this case, we expected edge grouping to cause propagation of local figural edge assignment from the biased section's dividing edge to the unbiased-section's dividing edge. On the other hand, these edges were clearly ungrouped when they moved at different rates and thus no longer shared a common fate (Figures 1D-1E), or collinearity (except at the instant when they "crossed"). In this edge-ungrouped case, we expected that propagation of figural edge assignment from the biased to the unbiased section would become minimal or absent. In subsequent experiments (see below), we used displays with static figure-ground cues instead, imposing other grouping manipulations, to test the generality of our findings and the new principle that they suggest.

Orthogonally to the edge-grouping manipulation described above, for completeness, we also manipulated 

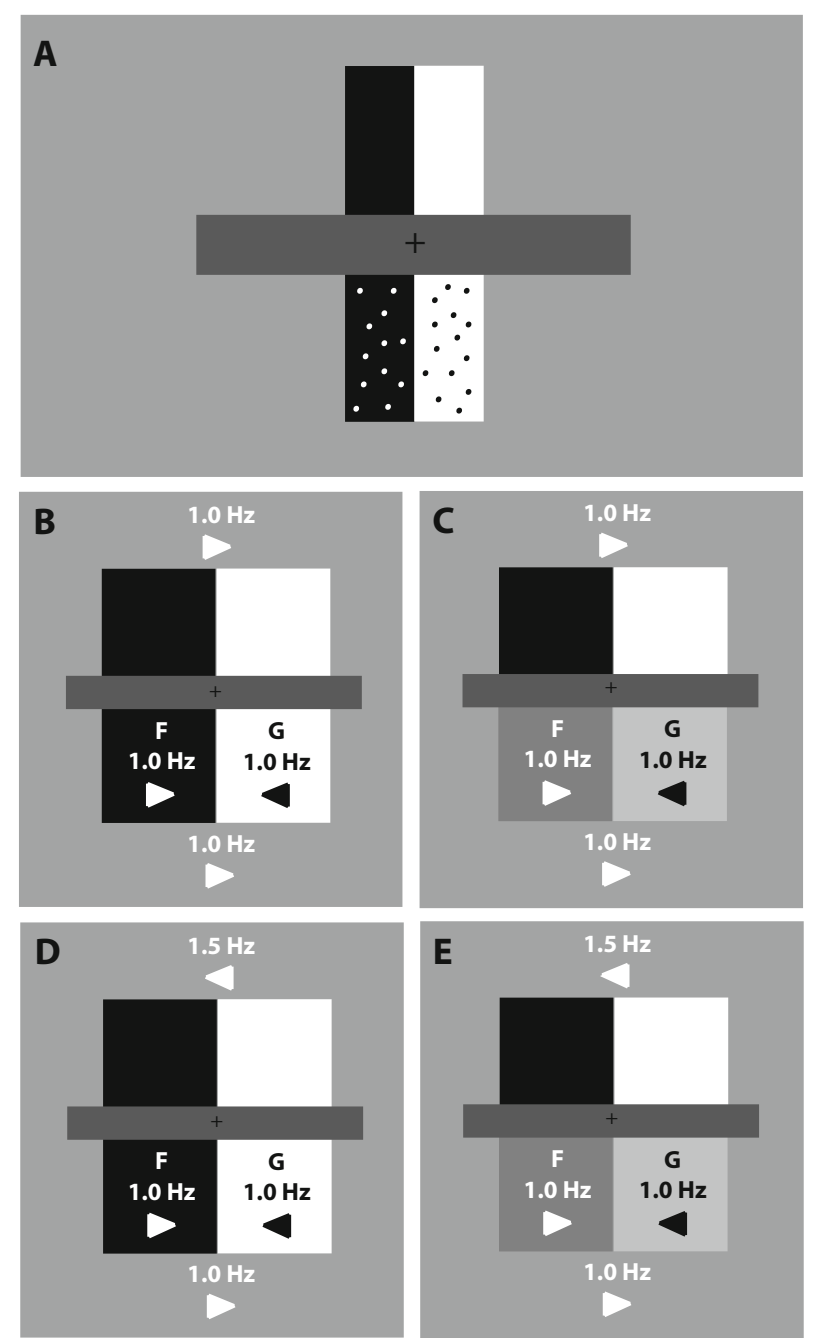

Figure 1. (A) Example static frame from the dynamic displays in Experiment 1A. The top (above rectangular occluder) blackand-white section is the unbiased section (no dots) in this example, whereas the bottom section (below rectangular occluder) is the biased section with texture dots on both sides of the edge. Edge assignment for the edge within the biased section was determined by local edge-assignment cues - namely, the relationship between the motion of the edge and the dots in the two regions (see main text). The color of the central "occluding" horizontal bar was red in the actual displays. Animated color versions of all of the displays are available in the supplemental materials. (B) Annotated cartoon of the motion for different sections of the display in the edges-grouped, region-color-similar conditions. Arrows within a region indicate the relative direction of motion for dotted region texture. Arrows above or below a section indicate the relative motion direction of the vertical dividing edge for that section. The frequencies listed indicate the rate of oscillation associated with that display element (e.g., "1.0 Hz" above a region arrow indicates a $1.0-\mathrm{Hz}$ oscillation for texture dots in that region). " $F$ " and " $G$ " indicate anticipated figural or ground status (respectively) within the biased section due to dot motion in relation to edge motion, as was confirmed by phenomenal judgments for the biased section. (C) Edge-only grouped condition, region color dissimilar. (D) Edges-ungrouped, regioncolor-similar conditions. (E) Edges-ungrouped, region-colordissimilar conditions. Blue and green region colors are replaced by dark gray and light gray here. In the actual displays, the blue and green were equiluminant (by flicker photometry for each participant) with the "black" (dark gray) and "white" (light gray) of the unbiased section, respectively. region-grouping by color similarity (henceforth termed region color similarity) in Experiment 1. Analogously to edge grouping, when the regional colors of the biased and unbiased sections were matched (i.e., region grouped by color similarity), we anticipated that figural edge assignment for the central dividing edge might be more likely to propagate between the two sections than when they had differently colored regions. Regions on either side of the critical vertical edges in both the biased and unbiased sections shared either region color similarity (i.e., both sections were black at left and white at right, or vice versa, in Experiment 1; Figures 1B and 1D), or else they did not (unbiased-section regions were black and white, whereas the biased-section regions were blue and green, or vice versa in Experiment 1; Figures 1C and 1E). Manipulating edge grouping and region color similarity orthogonally allowed us to assess whether only edge grouping (initially manipulated by common fate and collinearity, but see our later experiments also) affects figural edge-assignment propagation, or whether region color similarity also matters, as well as whether these factors interact.

\section{EXPERIMENT 1 \\ Dynamic Edge-Grouping Cues With Subjective Reports of Figural Assignment}

In Experiment 1, we used a subjective-report procedure to measure participants' phenomenal experience of figural edge assignment for the biased or unbiased sections of dynamic displays. On some trials, we measured the participants' perception of the biased section in order to determine whether local cues were effective in determining figural edge assignment, as was expected along the dividing edge in that section. In other trials, we measured figural edge assignment for the unbiased section's dividing edge instead. This allowed us to assess whether edge assignment propagated from the biased section's edge to the unbiased section's edge. We manipulated the edge-grouping and region-color-similarity factors as were described above in order to determine whether perceptual grouping affected propagation of subjective figural edge assignment from the biased to the unbiased dividing edge.

\section{Method}

Participants. Twelve right-handed people from the local community participated ( 6 male and 6 female; mean age $=21.4$ years) . All of the participants reported normal vision and gave informed consent to participate in the study.

Displays and Design. Each display comprised three sections (see five examples of alternative three-section displays in Figure 1): the top bipartite section, the bottom bipartite section, and the rectangular occluder between them. The top and bottom bipartite sections were each $5.3^{\circ}$ square and were separated by a red rectangle $\left(16.8^{\circ} \times\right.$ $2.0^{\circ}$ ), centered at fixation. The vertical dividing edges within each bipartite section oscillated horizontally (the oscillation distance was $0.85^{\circ}$ ) at either $1.0 \mathrm{~Hz}$ or $1.5 \mathrm{~Hz}$.

Edge assignment for the biased section's dividing edge was determined by powerful local cues to figural edge assignment (Palmer \& Brooks, 2008; Yonas, Craton, \& Thompson, 1987). Biased section regions on both sides of its dividing edge were filled with a sparse dot texture (Figure 1A, bottom). The dot texture on one side of the edge moved with the dividing edge, which should have assigned the 
edge to that region figurally (see Palmer \& Brooks, 2008). The dot texture on the other side moved in counterphase to the edge, so that region should have become ground.

When the biased and unbiased sections were related in terms of region color similarity, the regions in both sections were black (average $9.0 \mathrm{~cd} / \mathrm{m}^{2}$, herein termed black) and white (average $53.5 \mathrm{~cd} / \mathrm{m}^{2}$, herein termed white), with corresponding left-right assignments for colors within the two sections. The exact luminance for each color was determined for each participant individually by flicker photometry (Wagner \& Boynton, 1972). White was made equiluminant to green, and black was made equiluminant to blue. This was done to ensure that the region colors were similar in luminance to the green and blue colors used in the region-color-dissimilar conditions. In the region-color-dissimilar conditions, biased-section regions were green $\left(53 \mathrm{~cd} / \mathrm{m}^{2}\right)$ and blue $\left(8 \mathrm{~cd} / \mathrm{m}^{2}\right)$.

The design was a $2 \times 2$ within-subjects factorial design, with edge grouping and region color similarity as the factors. There were 32 repetitions of each of the four conditions within each block. Several other aspects of the displays were counterbalanced. In the biased section, the dot-motion-determined figure was either on the left or right side of the vertical edge, equiprobably. Likewise, the initial direction of motion for the biased section's dividing edge was either leftward or rightward equally often. This counterbalanced for the effects of the recently described figure-ground cue of advancing versus receding motion (Barenholtz \& Tarr, 2009) that, in the present study, could have arrived in the form of initial expansion versus contraction for either side of the moving dividing edge, from display onset. The biased section was equally often above or below the occluder. In edge-grouped conditions, the initial direction of motion for the biased section's edge was the same as that for the unbiased section, whereas it began with opposite motion in edge-ungrouped conditions. Edge oscillation frequency in the biased section was either 1.0 or $1.5 \mathrm{~Hz}$, equiprobably. In edge-grouped conditions, the frequency was the same as that for the unbiased section, whereas for edge-ungrouped conditions, it was different. The color of the two regions within the unbiased section was counterbalanced (white on the left or right), and the color in the biased section was equiprobably either the same (in region-color-similar conditions) or differed in being a green/blue combination (region-color-dissimilar conditions). In the latter case, contrast polarity across the edge (i.e., dark/ light or light/dark) was counterbalanced by using either blue/green (on the left or right, respectively) or green/blue (on the left or right, respectively) instead.

Participants reported phenomenal figure-ground assignment for only one section of the display (upper or lower) in each block. Because the judged section was equiprobably biased or unbiased, there were two separate sets of results. Judgments of the biased section allowed us to confirm whether the local figure-ground bias that was induced by the dots was indeed effective. Judgments of the unbiased section provided the critical new test for any propagation of figural edge assignment from the biased section to the unbiased section.

Procedure. Displays were presented on a 21 -in. CRT computer monitor $(60 \mathrm{~Hz}, 1,280 \times 1,024$ pixel resolution) using Presentation software (Neurobehavioral Systems, Inc., www.neurobs.com). Each trial began with a central fixation cross for $1,000 \mathrm{msec}$, followed by the 2,800-msec display. Participants reported, for just one section of the display (top or bottom, blocked) whether the left or right region within that section appeared to be "in front," using corresponding buttons. Because the judged section was equally often biased or unbiased, there were two sets of results: biased-section judgments (assessing whether the local dot-motion biased that section's figural assignment, as expected) and unbiased-section judgments (assessing any propagation from the biased section). Eye position was monitored throughout displays using an ASL-5000 Tracker (Applied Science Laboratories, Inc.; Bedford, MA, USA; www.a-s-1.com). Trials with $>1^{\circ}$ deviations from fixation or with pursuit eye movements $\left(>0.5^{\circ}\right.$ regular oscillatory structure) during the displays were ex- cluded $(0.9 \%)$. The procedure was approved by the UCL Psychology Department Ethics Committee.

\section{Results and Discussion}

As expected for the biased section, participants primarily reported as figural ("in front") the side on which the dot texture moved together with the dividing edge. They selected this for $94.7 \%$ of judgments on the biased section - significantly greater than 50\% $(t=23.31, p<$ $.001)$. This was as expected, in accord with the local figural edge-assignment cues's favoring that region (Palmer $\&$ Brooks, 2008; Yonas et al., 1987). There were no significant effects of any of the counterbalanced factors on biased-section figure-ground judgments. At the request of a reviewer, we assessed any impact from the starting direction of motion for the dividing edge, which made one side of the section expand and the other contract (Barenholtz \& Tarr, 2009) before the motion reversed. Participants chose the initially expanding region $50.3 \%$ of the time - no greater than chance $[t(11)=1.86, p<$ $.09]$. The lack of a significant impact from this initial expansion/contraction in the present study might be due to the much stronger impact of our intended figural edge-assignment cue for the biased section (due to how the dots moved in relation to the dividing edge; see Palmer \& Brooks, 2008). That dot-motion factor determined figure-ground assignment $94.7 \%$ of the time for the biased section.

For our critical new test of whether figural assignment propagated from the biased section to the unbiased section, the unbiased-section judgments were recoded as either context consistent (e.g., when the biased section's edge should have been assigned leftward via the dots, participants correspondingly reported leftward assignment for the unbiased section), or context inconsistent. Figure 2 plots the percentage of context-consistent responses in the four main conditions. These context-consistent responses were significantly higher when the edges were grouped $[F(1,11)=63.36, p<.0001]$ and when region color similarity was present $[F(1,11)=8.27, p<.015]$. These results indicate that figural edge assignment propagated from the biased to the unbiased section, but only when grouping between these two sections was present. In addition to the main effects, the two factors also interacted $[F(1,11)=4.96, p<.04]$. This interaction reflects an impact of region color similarity only when edge grouping was present $[F(1,11)=16.04, p<.002]$, not when the two critical vertical edges were ungrouped $(p=.46)$.

The percentage of context-consistent responses for the unbiased section was significantly greater than $50 \%$ in the edge-grouped conditions (for edge-and-region-grouped conditions, $t=11.22, p<.001$; for edge-only-grouped conditions, $t=8.11, p<.001$ ), but not in the edgeungrouped conditions (region-only-grouped conditions, $p=.64$; neither-grouped conditions, $p=.87$ ). For completeness, we report also that the counterbalanced factor of edge-contrast similarity (i.e., same or different contrast polarity across edges in biased and unbiased sections, which was varied intrinsically but only for region-color- 


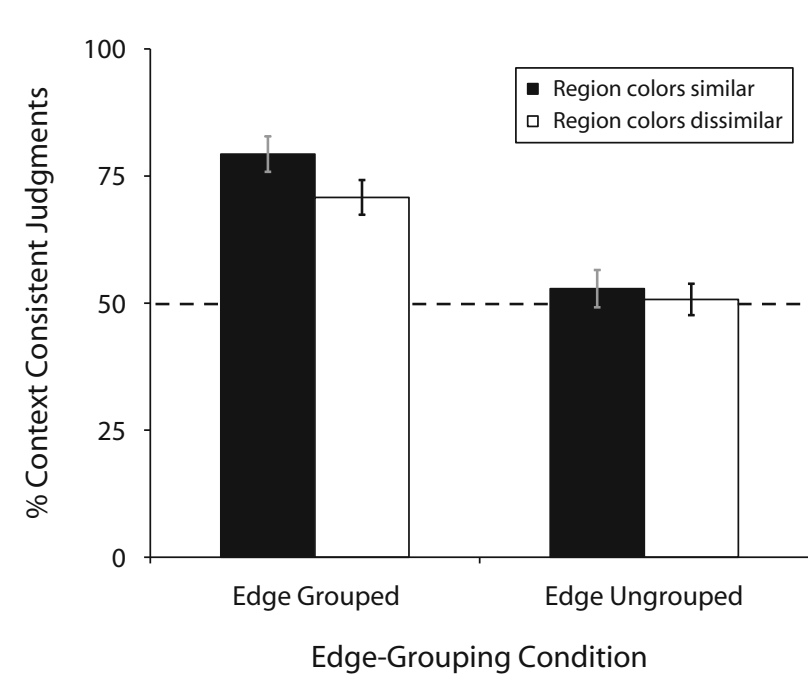

Figure 2. Results of Experiment 1. The average percentage of trials on which the context-consistent side within the unbiased section of the display was chosen as figural (i.e., in accord with the side biased as figural in the separate biased section) is plotted as a function of edge-grouping and region-color-similarity factors. Error bars represent $\mathbf{9 5 \%}$ within-subjects confidence intervals. Note the higher context-consistent responses in the edge-grouped condition, with this slightly increasing when regional color is also similar.

dissimilar conditions) showed a very small yet reliable influence [same $61 \%$ vs. different $59 \% ; F(1,11)=5.21$, $p<.043]$.

Finally, a reviewer asked us to examine any impact of the expansion/contraction on one or the other side of the dividing edge, to its initial direction of motion. The initially expanding side within the unbiased section was chosen as figural $59.5 \%$ of the time (in accord with Barenholtz \& Tarr, 2009), slightly but significantly above chance $[t(11)=8.48, p<.001]$. This effect for the unbiasedsection judgments (that was not significant for the biased section) might reflect different strengths of figure-ground assignment for the two sections. Within the unbiased section, there were no local cues to edge assignment, and the propagated contextual effect that we uncover here for the first time (see Figure 2) did not reach ceiling levels. This might have left some room for modulation by other figure-ground cues, such as expansion/contraction.

The critical new aspect of our results lies in showing that subjective figural edge assignment of a locally unbiased edge can be affected by the figural edge assignment of a remote contextual biased edge. Moreover, this assignment propagates from biased to unbiased edges only when they are grouped together (here by common fate and collinearity, but see our later experiments for different grouping cues). Region color similarity had some influence, but only when it was not vetoed by edge ungrouping. Same or different edge-contrast-polarity also had a minor influence, but was only varied in a minor way in Experiment 1 - that is, only for the region-colordissimilar conditions, since contrast polarity had not been of a priori interest to us.

\section{EXPERIMENT 2 \\ Control for Salient Dots Potentially \\ Attracting Attention When Figural Assignment Is Measured by Subjective Report}

In Experiment 1, we demonstrated that figural edge assignment along an edge with no local cues to edge assignment (e.g., our unbiased-section edge) can be determined by edge assignment along an edge elsewhere in the image (i.e., our biased-section edge), provided that this contextual edge is grouped with the unbiased edge. Our interpretation of this finding is that perceptual grouping can gate propagation of figural edge assignment between remote parts of an image (here, the biased and unbiased sections). In Experiment 1, the local edge-assignment cues within the biased section involved moving dots. These dots were presented on both sides of the dividing edge in the biased section (see Figure 1A), but the dots on one side moved together with the central edge (see the supplemental materials animations), causing that side to become figural, in line with previous results (Palmer \& Brooks, 2008; Yonas et al., 1987). Dots on the other side of the biased section moved in counterphase to the dividing edge and thus caused that side to be perceived as ground. Thus, in our initial conditions, regions on both sides of the biased edge had moving dots. Nevertheless, one might still potentially be concerned that attention could be drawn to salient moving dots (Franconeri \& Simons, 2005; Hillstrom \& Yantis, 1994), perhaps more so on the figural side. Furthermore, there have been some suggestions that familiar figural regions may attract attention (Nelson \& Palmer, 2007), although it is unclear whether nonfamiliar regions (like those used in the present study) can attract attention by their figural status alone - a point we will return to in the General Discussion. Returning to the interpretation of the present Experiment 1, if the dots on one side indeed became more salient and thereby attracted attention, one might then, in principle, argue that it could be attention toward the side of more salient dots in the biased section, rather than figural assignment, per se, that ultimately leads to an influence on the unbiased section. This might conceivably occur, for instance, via object-based or grouping-related attention mechanisms (see, e.g., Egly, Driver, \& Rafal, 1994) that might conceivably allow attention to spread to the unbiased section.

To address this concern for the specific issue of salient dots potentially attracting attention toward one side, in Experiment 2 , we used the dots within the biased section's regions to manipulate which side of the dividing edge should be most "salient" (in terms of containing moving dots) within that biased section, separately from which region should be figural. In some displays, we now had moving dots present on only one side of the biased section, rather than on both. This was particularly telling when the dots appeared only on the ground side in the biased section (henceforth, termed the ground-salient condition). In this case, the dots move in counterphase to the dividing edge's motion and thus should still make that region appear as the ground, leading to the figural assignment of the dividing 
edge to the other region (with no dots). But any account in terms of attention capture by salient moving dots should presumably then clearly favor the biased section's ground side (now containing the only moving dots) rather than the anticipated figural side (now with none). For an account solely in terms of attention's being attracted to salient moving dots (see also our General Discussion section for consideration of further accounts), this should lead to the opposite prediction of that expected from our own perspective, in terms of grouping-mediated propagation of edge assignment.

We compared the ground-salient condition with a condition with dots on both sides (salience-balanced, the same as the stimuli in Experiment 1), and also for completeness with a condition with the moving dot texture appearing on only the biased section's figural side (figure-salient). If our grouping-mediating propagation of figural edge assignment account is correct, then the pattern of results should be similar for all three conditions. In particular, having dots present only on the ground side of the biased section should not reverse the pattern of results for the unbiased section, provided that the expected figural assignment still applies for the biased section. By contrast, if attention capture by salient moving dots is critical, then the ground-salient condition in particular should lead to a reversed outcome for the unbiased section.

\section{Method}

Participants. Sixteen people from the local community participated ( 7 male, 9 female, average age $=26.1$ years). All reported normal vision and gave informed consent. None of these participants had taken part in Experiment 1.

Displays and Design. In the salience-balanced conditions, the displays were exactly as they were in Experiment 1 . In the groundsalient condition, the displays were as for those in Experiment 1, except that the moving dots on the figural side of the biased section were now removed so that only those moving in counterphase to the edge (in the ground side) remained. In the figure-salient condition, the displays were the same as those for Experiment 1, except that the moving dots on the ground side of the biased section were removed, so that only those moving in common fate with the edge (on the figural side) remained. We note in passing that having moving dots only on the figural side might have slightly reduced the strength of figural assignment by the dots (since this condition removes the dots that move in counterphase to the dividing edge). By contrast, in terms of possible attention capture, the figure-salient condition should presumably instead act only to increase any effects. All other aspects of the displays were identical to those in Experiment 1.

The design was a $2 \times 2 \times 3$ within-subjects factorial design. The first two factors were the same edge-grouping and region-colorsimilarity factors as in Experiment 1. The third factor specified the salient side due to the dots: figure salient, ground salient, or salience balanced (only the salience balanced had dots on both sides of the dividing edge in the biased section, equivalent to that in Experiment 1). The counterbalancing of other display factors was the same as in Experiment 1. There were 32 repeated measures per condition within each block.

Procedure. The procedure was the same as in Experiment 1. In one block, the participant judged the top section, and in the other block, he or she judged the lower section. There were four breaks in each block. Trials with $>1^{\circ}$ deviations from fixation or with pursuit eye movements $\left(>0.5^{\circ}\right.$ regular oscillatory structure) during the displays were excluded (intersubject mean of $1.8 \%$ trials excluded).

\section{Results and Discussion}

The local edge-assignment cues due to the dot motion within the biased section were effective (significantly greater than $50 \%$ ) at determining edge assignment within that section in all three dot-salience conditions: figuresalient, $87.2 \%[t(15)=17.11, p<.0001]$; ground-salient, $97.9 \%[t(15)=96.23, p<.0001] ;$ salience-balanced, $99.2 \%[t(15)=181.64, p<.0001]$. Notably the local edge-assignment cue from dot motion remained equally effective in the balanced-salience and ground-salient conditions. Its influence was somewhat reduced in the figuresalient condition [significantly so in comparison with either of the other two dot conditions; for both, $t(15)>4.9$, $p<.001]$, but still remained significant. As was briefly anticipated in the introduction to this experiment, this reduction may have occurred because (contrary to an account solely in terms of attention capture by salient dots) the counterphase motion of dots on the ground side may provide a particularly strong figural-assignment depth cue (Palmer \& Brooks, 2008) when present.

As for Experiment 1, at the request of a reviewer, we also analyzed any impact from the counterbalanced cue of initial expansion versus contraction on one or the other side of the dividing edge in the biased section, due to its initial direction of motion before the oscillatory reversal. We did this separately for each of the dot salience conditions. In the salience-balanced condition, the initially expanding region was chosen $50.1 \%$ of the time-no greater than chance $[t(15)=0.56, p<.58]$. In the ground-salient condition, the initially expanding region was chosen $50.6 \%$ of the time-again, no greater than chance $[t(15)=1.21$, $p<.24]$. The results in these two conditions are consistent with Experiment 1's biased-section results. In contrast, there was a small but significant effect of initial expansion versus contraction for biased-section judgments in the figure-salient condition $[t(15)=2.23, p<.04]$, with the initially expanding side selected on $52.6 \%$ of trials. We note that this same figure-salient condition showed slightly less impact of the local dot-motion figure-ground cue (presumably due to the removal of dots moving in counterphase to the dividing edge), so it was not as close to ceiling as the other two conditions. This might have led to more sensitivity to any impact from expansion/contraction at onset (see also our Discussion section of Experiment 1), although we note that expansion/contraction was fully counterbalanced with respect to the main factors of interest here, so it cannot explain any of our critical results.

We turn next to the critical judgments for the unbiased section (Figure 3) that allow us to measure any propagation of figural assignment from the biased section. In all salience conditions, there was always an impact of edge grouping [for salience balanced, $F(1,15)=201.05, p<$ .0001 ; ground salient, $F(1,15)=285.53, p<.0001$; and figure salient, $F(1,15)=102.20, p<.0001]$. There were also main effects of region color similarity [for salience balanced, $F(1,15)=4.7, p<.047$; ground salient, $F(1,15)=5.43, p<.034$; figure salient, $F(1,15)=5.94$, $p<.028$ ]; plus the usual interaction between edge grouping and region color similarity [for salience balanced, 


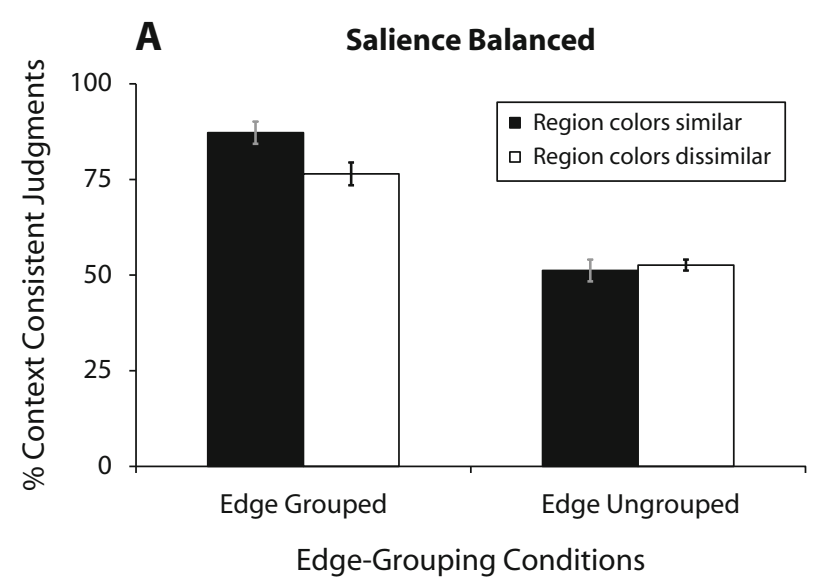

B

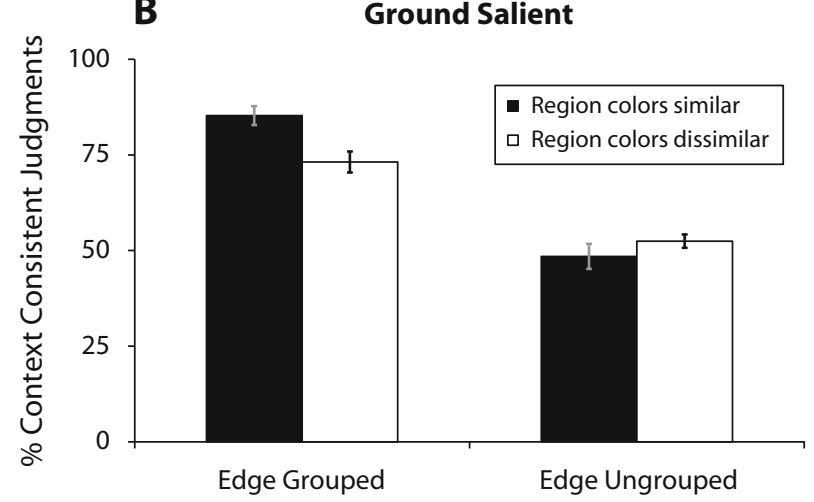

Edge-Grouping Conditions

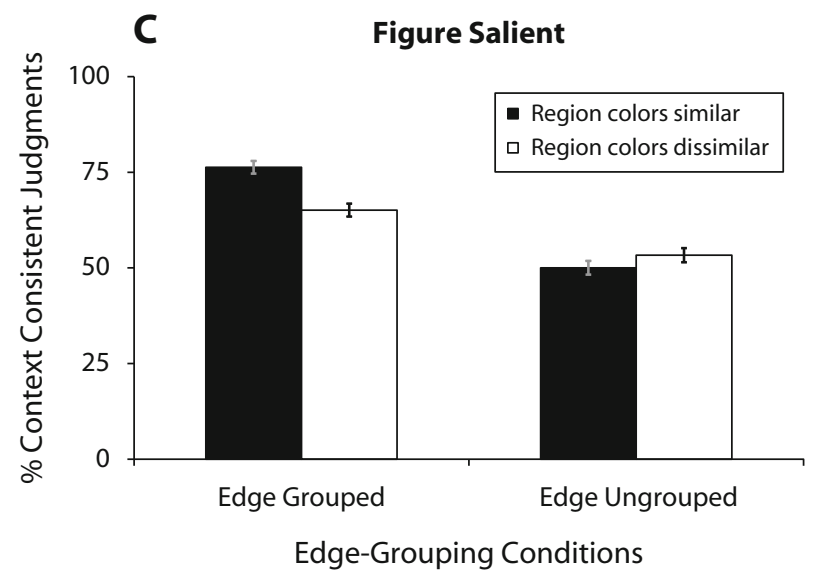

Figure 3. Results of Experiment 2. (A) Results for the saliencebalanced condition (i.e., moving dots on both sides in the biased section). (B) Results for the ground-salient condition (i.e., moving dots now only on the ground side in the biased section). (C) Results for the figure-salient condition (moving dots now only on figural side in the biased section). Error bars represent $95 \%$ withinsubjects confidence intervals. Note the similar outcomes in all three salience conditions - namely, higher context-consistent responses in the edge-grouped condition, with this slightly increasing when regional color is also similar.

$F(1,15)=9.56, p<.007$; ground salient, $F(1,15)=$ $10.69, p<.005$; figure salient, $F(1,15)=41.03, p<$ $.0001]$. These interactions all arose because (as in Experi- ment 1 also; compare Figures 2 and 3) region color similarity had an impact only when the edges were grouped. The overall $2 \times 2 \times 3$ within-subjects ANOVA showed main effects of edge grouping $[F(1,15)=441.96, p<$ $.0001]$, region color similarity $[F(1,15)=11.56, p<$ $.004]$, and the interaction of these two factors $[F(1,15)=$ $36.21, p<.0001]$. For all of the three dot-salience conditions, the aforementioned statistics confirm that the basic pattern of results we saw in Experiment 1 (i.e., that edge assignment propagates from the biased to the unbiased edge when edge grouping is present, and is further facilitated by additional region grouping) is still found, even with the moving dots on just one or the other side of the dividing edge in the biased section, rather than on both sides. Most notably, the pattern of results for the unbiased section was unchanged when removing the dots on the figural side of the biased section, even though in terms of putative attention capture by salient moving dots, that situation might have been expected to reverse the outcome for the unbiased section.

As was noted above, there was a small effect of dot salience on the strength of the local cue within the biasedsection (i.e., this local cue was somewhat weaker in the figure-salient condition, presumably due to the lack of any counterphase dot motion). Given that figural edge assignment in the biased section must be present in order to propagate to the unbiased section, it follows that any changes in the strength of the local cue for the biased section may also propagate through to the unbiased section (provided, of course, that edge grouping and region grouping are present to allow such propagation). Consistent with this, there was a main effect of dot salience for the biased section results $[F(2,30)=6.17, p<.006]$, reflecting somewhat lower context-consistent responses in the figure-salient condition. Moreover, consistent with our account, this reduction was most apparent in the edgegrouped conditions, leading to an interaction with edgegrouping $[F(2,15)=11.29, p<.0001]$. There was no higher level three-way interaction $(p>.75)$.

As in the unbiased-section judgments of Experiment 1, we also found a small but reliable effect of the initial expansion/contraction cue. Participants chose the initially expanding region as figure significantly more than $50 \%$ in all three dot-salience conditions: salience balanced, 52.9\% $[t(15)=2.47, p<.02]$; figure salient, $54.3 \%[t(15)=2.36$, $p<.03]$; ground salient, $55.2 \%[t(15)=3.45, p<.004]$. We note once again that this initial expansion/contraction factor (determined by the initial direction of motion of the dividing edge, prior to the oscillatory change in direction) was fully counterbalanced with respect to our main factors of interest, and that it therefore cannot explain the effects of the latter factors.

Figure 3 displays a total of three replications of our key findings from Experiment 1 (cf. Figure 2). Once again, figural edge assignment of the biased edge propagated to the unbiased edge only when these edges were grouped (in the present study by common fate and collinearity, but see our later experiments for other grouping cues), whereas region color similarity exerted a small but reliable influence on such propagation only when the edges 
were grouped. Most importantly, all of this still occurred in the ground-salient condition, in which the salient dots should, if anything, presumably attract attention toward the ground rather than toward the figural region of the biased section. If such attention capture by moving dots in itself mediated the spread of figural edge assignment, then one should expect the effects to reverse in the groundsalient condition because the presence of moving dots should now favor the side opposite to the figural side. But, in fact, the effects were just as strong in the ground-salient condition as they were in the balanced-salience condition (which further replicated Experiment 1).

In the figure-salient condition, context-consistent responses were slightly lower, but this was in accord with the slightly weakened local impact of the dot-motion cue within the biased section of those particular displays (which now had no dots in counterphase to the dividing edge of the biased section). Nevertheless, even the (correspondingly) slightly weakened impact on the unbiased section in this situation still showed a similar overall pattern to the other salience conditions (see Figure 3). Once again, figural edge assignment propagated to the unbiased section's edge when edge grouping was present between the dividing edges in the two sections, with this effect being modulated by region color similarity, but only in the presence of edge grouping.

\section{EXPERIMENT 3}

\section{Objective Short-Term Edge Matching As an Index for the Consequences of Figural Assignment}

In Experiment 3, we sought to generalize our key findings to an objective consequence of edge assignment, rather than for phenomenal judgments, as studied in Experiments 1 and 2. Driver and Baylis (1996) introduced a short-term edge-matching (STEM) procedure that reflects figural edge assignment. Their participants judged whether a curvy edge in a "probe" display matched the curvy dividing edge in a preceding "prime" display. Performance was better if the probe display's edge had the same figural edge assignment as in the preceding prime display, as opposed to when a "ground" probe was used instead (although in either case, equivalent curvy edges were presented).

We adapted this STEM procedure in the present study so that participants now saw a prime like the displays in our Experiment 1, but the unbiased section's dividing edge was now curvy rather than straight (Figure 4A). Each prime was followed by either of the two types of probepair displays (see Figure 4B or 4C), each containing two shapes with curvy edges. For both types, one of the curvy probe edges matched the curvy edge in the unbiased section of the preceding prime display. The other was a previously unseen foil. The objective task was to select the matching probe. Each probe pair comprised either "figure" probes (i.e., with their curvy edges assigned in the same direction as the edge in the preceding prime biased section), or "ground" probes, both with edges assigned to the other side. The task was to pick whether the upper or
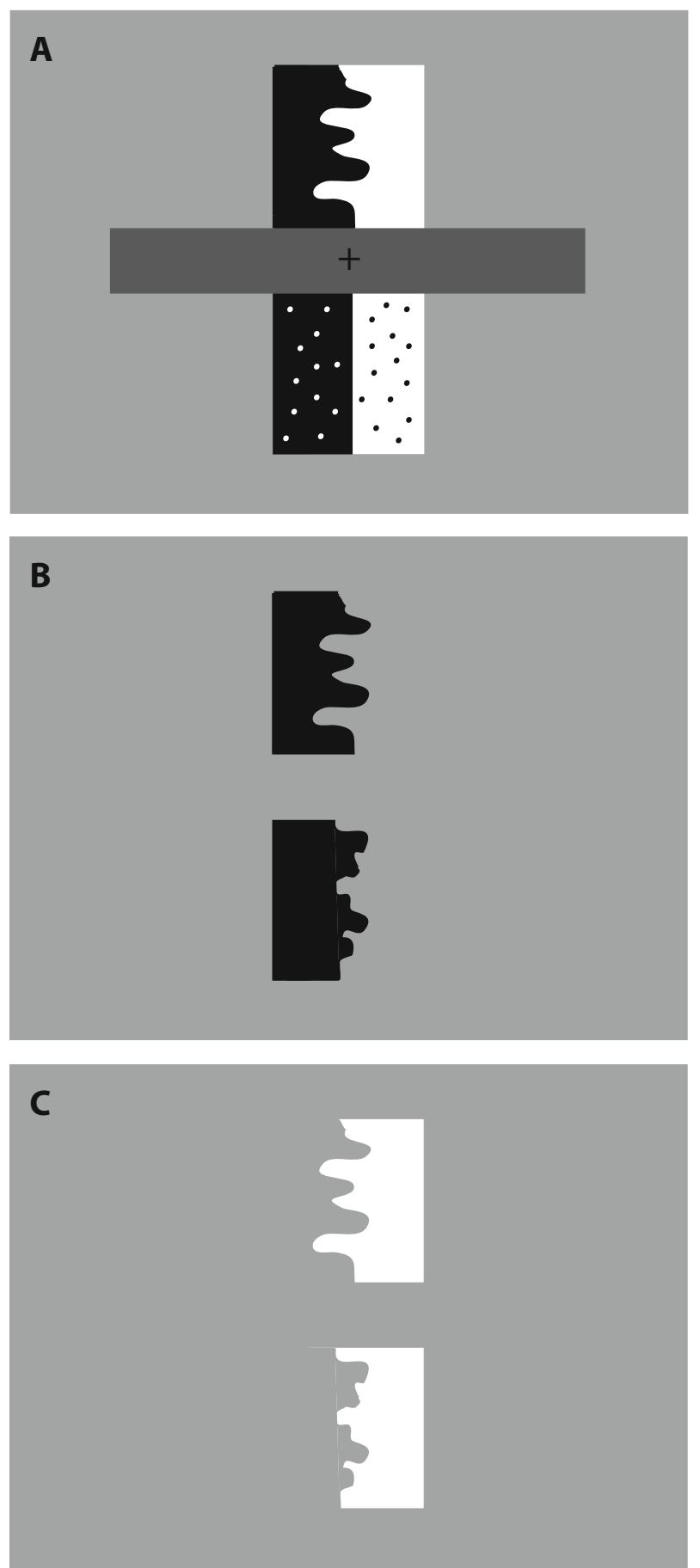

Figure 4. Example displays from Experiment 3 for the STEM task; see main text for description of that task. (A) Prime display shown with curved dividing edge in the upper unbiased section. (B) "Figural" probes, assuming that the left side was defined (by the biased section's moving dots) as figural in preceding prime. The top probe shape in panel $B$ matches the prime curvy edge in panel A. (C) "Ground" probes assuming that the left side was defined as figural in the preceding prime. The top probe shape in panel $\mathbf{C}$ matches the prime curvy edge in panel $\mathrm{A}$.

lower edge in a given probe display had the same curvy edge as the preceding unbiased prime. This now allowed us to test for objective consequences (rather than subjec- 
tive reports; see Experiments 1 and 2) of our grouping manipulations on the propagation of figural edge assignment from the biased section to the locally unbiased section of the prime display. Under the appropriate grouping conditions for the prime displays, figure probes should lead to more efficient matching performance than ground probes (analogously to Driver \& Baylis, 1996), if figural edge assignment does indeed propagate from biased to unbiased sections when grouped, and if this has objective consequences for edge assignment. If figural edge assignment does not propagate to the unbiased section, then we should see no difference between figure and ground probe performance in any of the conditions.

\section{Method}

Participants. Sixteen people from the local community participated ( 6 male, 10 female; average age $=28.9$ years). All reported normal vision and gave informed consent. None of these participants had taken part in Experiments 1 or 2.

Displays and Design. The STEM task prime displays were similar to the Experiment 1 displays, although instead of a straight dividing edge, the unbiased section now had a unique curvy edge on each trial, with no local edge-assignment cues. The curvy edges were generated by a pseudorandom algorithm that divided each edge into a set of vertical segments that were of 10-30 pixels in length. Each segment was then randomly assigned a horizontal deviation from the vertical midline of the box that could range from 0 to 45 pixels in either direction from the horizontal center of the box. The top and bottom segments were constrained to have a horizontal deviation of 0 . The total area of the regions to the left and the right side of the edge was then approximately equated by displacing all of the edge segments (except the top and bottom) an equal amount in the required direction. The resulting edge was then smoothed with a 5-pixel-wide averaging kernel. The overall convexity of the regions was approximately equated by ensuring that the horizontal deviations occurred equally often to the left and right of the horizontal center. The same set of edges was used for each participant. However, these edges were randomly assigned to conditions separately for each participant. The luminance values of the black and white regions were $8.8 \mathrm{~cd} / \mathrm{m}^{2}$ and $53.3 \mathrm{~cd} / \mathrm{m}^{2}$, respectively.

Probe displays always comprised two shapes (each $3.97^{\circ}$ horizontally $\times 5.3^{\circ}$ ). The curvy edge of one shape exactly matched the preceding prime display's curvy edge, whereas the other (foil) was a new, unique curvy edge. Matching and foil edges in figure probes were each assigned (by closure/surroundedness; see Figures 4B and $4 \mathrm{C}$ ) to the same side as the biased section's straight edge in the preceding prime display. Ground probe curvy edges were each assigned oppositely. Contrast polarity across the probes' curvy edge was always the same as in the prime display (see Figure 4). Curvy edges were aligned with the screen's horizontal meridian and were centered $5.3^{\circ}$ vertically above and below fixation. The matching probe within each pair was equiprobably above or below fixation. The top or bottom location for the unbiased section in the prime display was now counterbalanced between subjects rather than within subjects as had been done in Experiments 1 and 2. The type of probe (figure or ground, with respect to the preceding biased section) was an additional within-subjects factor in this design. All other display counterbalancing was the same as that for Experiment 1 . There were 32 repeated measures per condition within each block. Two blocks were presented.

Procedure. Trials began with a 1,000-msec fixation cross, followed by the 2,800 -msec prime display, a 500 -msec prime-probe interstimulus interval (ISI), and, finally, the probe display until response. Participants judged which curvy probe edge (upper or lower) matched the prime display's curvy edge, using a mouse button. Par- ticipants were instructed to respond as quickly as possible without sacrificing accuracy. They were given 40 practice trials before the experimental trials began. Trials with $>1^{\circ}$ deviations from fixation or with pursuit eye movements ( $>0.5^{\circ}$ regular oscillatory structure) during the prime displays were excluded (the intersubject mean was $0.85 \%$ of trials excluded).

\section{Results and Discussion}

Because the pattern of results was the same for STEM error and reaction time (RT) measures, the two measures were combined into an inverse efficiency (IE) score (mean correct RT divided by accuracy rate) to simplify presentation and analysis (Townsend \& Ashby, 1978). See the supplemental materials for error and RT results, separately. IE scores (Figure 5) showed main effects of probe type $[F(1,15)=23.08, p<.0001]$ and edge grouping $[F(1,15)=11.49, p<.004]$. There were interactions of probe type and edge grouping $[F(1,15)=26.37$, $p<.0001]$ and probe type and region color similarity $[F(1,15)=12.74, p<.003]$, and a probe $\times$ edge grouping $\times$ region color similarity $[F(1,15)=4.81, p<.04]$. These interactions show that performance was better for figure than for ground probes when edge grouping and region color similarity were present $[t(15)=7.52, p<$ $.0001]$, or in the presence of edge grouping only $[t(15)=$ $4.91, p<.0001]$, but that region color similarity had no effect when edges were ungrouped in the prime display (no effect for the region-color-similarity-only conditions $[t(15)=0.23, p<.82]$. There was no difference between figural and ground probes when neither edge grouping nor region color similarity was applied during the prime $[t(15)=0.93, p<.36]$. This lack of an effect when edge and region grouping were not present demonstrates once again that simply having a biased section elsewhere in the image is not sufficient to cause propagation of edge assignment. Rather, the biased and unbiased section edges must be grouped in order for edge assignment to propagate from the biased to the unbiased section.

Although providing a very different measure, the STEM results of Experiment 3 are perfectly analogous to those for the subjective reports in Experiment 1 (and in Experiment 2), in terms of the impact of the edge-grouping and region-color-similarity factors. These factors now affected performance in recognizing different types of probe shapes, rather than merely affecting subjective report, as before. As in Experiments 1 and 2, figural edge assignment propagated from the biased section's edge to the (now curvy) edge within the unbiased section, but only when those two sections were grouped by edge grouping. Because of this, the curvy edge in the unbiased section was then more likely to be seen as assigned in the corresponding direction. Hence, participants were more efficient (faster and more accurate; see the supplemental materials) at recognizing that edge in the probe phase when it was figurally assigned in the same direction for the probe as for the unbiased section in the prime. Also, as in Experiments 1 and 2, region color similarity had a small effect, but only when the edges were also grouped. These results provide a further replication of our effects that now demonstrate the consequences for objective performance. 


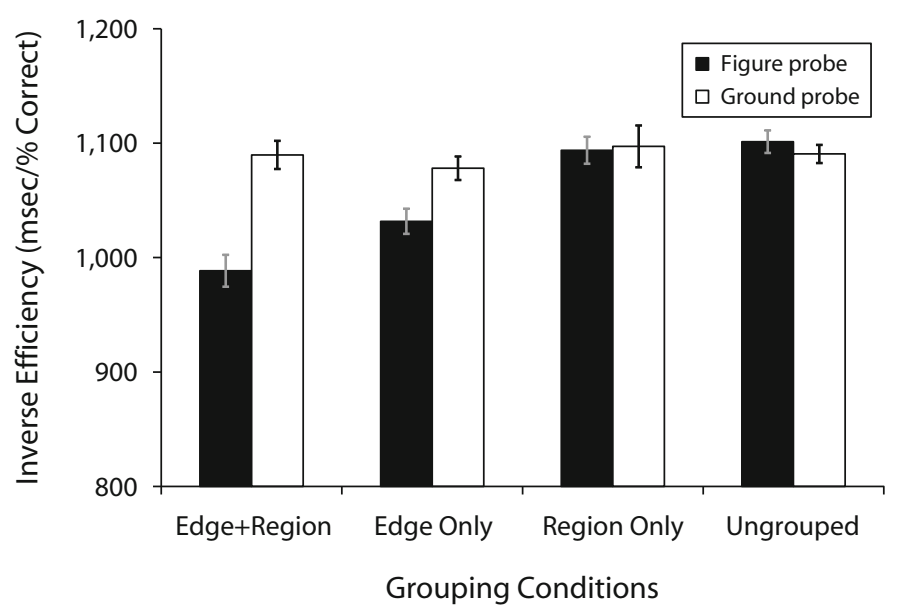

Figure 5. Results of Experiment 3. Average inverse efficiency (corresponding to the mean correct reaction time divided by the proportioncorrect responses) is plotted as a function of edge grouping, region color similarity, and probe type. Error bars represent $95 \%$ within-subjects confidence intervals. Note the enhanced efficiency of performance for the figure as compared with that for group probes when the edges were grouped (second pair of bars from left). The efficiency was even more pronounced when edges were grouped and regional colors were matched (leftmost pair of bars).

For completeness, at the request of a reviewer, we again tested further for any impact from the fully counterbalanced factor of which side of the unbiased section initially expanded (vs. contracted), due to the initial direction of motion of the dividing wavy edge, prior to the oscillatory reversal in direction. To assess this, we had to recode the data so that if, for instance, the left region of the prime display's unbiased section initially expanded (i.e., the wavy dividing edge moved rightward first), then a matching subsequent static probe edge that was assigned leftward would be recoded as a congruent probe. For this example, a matching edge that was assigned rightward instead in the static probe would be recoded as a potential incongruent probe. The opposite would apply if the right region of the unbiased section had expanded first during initial moving exposure. We found no impact of the initial expansion/ contraction factor on STEM performance [mean RT for congruent probes $(993.6 \mathrm{msec})$ and incongruent probes $(990.6 \mathrm{msec})$ did not differ, $t(15)=0.404, p=.602$, when the data were coded this way]. Error rates also showed no impact $[8.0 \%$ vs. $7.2 \%, t(15)=-1.16, p=.264]$. These results indicate that, unlike our more critical factors of interest, the counterbalanced initial expansion/contraction cue does not affect performance in the STEM task for the current context. This differs from some of the small but reliable impacts of this counterbalanced factor on subjective judgments in Experiments 1 and 2. This might reflect some potential differences between phenomenal versus objective measures, and/or perhaps greater sensitivity of the phenomenal measure to a subtle influence originating at initial display onset. The probes for the STEM task appeared 3,300 msec after initial onset of the prime displays, and subsequent to oscillatory motion in the primes, which meant that either side of the dividing edges had both undergone expansion and contraction by then.

Fortunately, these considerations of the fully counterbalanced expansion/contraction cue are all orthogonal to our main factors of interest, which showed in Experiment 3 that the key results of Experiments 1, in terms of grouping-constrained propagation of figural assignment, do extend also to an objective consequence of figural assignment, namely for the STEM task.

\section{EXPERIMENT 4 \\ Control for Salient Dots Potentially Attracting Attention When Using the STEM Task As the Measure for the Consequences of Figural Assignment}

Analogously to Experiment 2, we next manipulated the location of the moving dots (appearing on one or the other or both sides of the dividing edge in the biased section of the prime). This was in order to address the potential concern of the moving dots potentially attracting attention to one side, as was described in the introduction for Experiment 2 . We now verified the results using the objective STEM task, as per Experiment 3, rather than the subjective measure, as used in Experiment 2.

\section{Method}

Participants. Sixteen people from the local community ( 8 male, 8 female; average age $=23.4$ years) participated. All reported normal vision and gave informed consent. None had taken part in Experiments 1,2 , or 3 .

Displays and Design. The prime displays were the same as in Experiment 2. The parameters of the probe displays were the same as in Experiment 3. The counterbalancing of display parameters was 
the same as that for Experiment 2. Within each block, there were 32 repeated measures per condition in the $2 \times 2 \times 2 \times 3$ withinsubjects design. The factors were edge grouping (2), region color similarity (2), probe type (2), and salient dots location (3). Each participant completed two blocks. There were four breaks within each block.

Procedure. The procedure was the same as in Experiment 3, except for the further manipulation of salient dots location. Trials with $>1^{\circ}$ deviations from fixation or with pursuit eye movements $\left(>0.5^{\circ}\right.$ regular oscillatory structure) during the prime displays were excluded (intersubject mean of $1.3 \%$ trials excluded).

\section{Results and Discussion}

In the overall $2 \times 2 \times 2 \times 3$ ANOVA, there was a significant main effect of probe type $[F(1,15)=31.67$, $p<.0001]$, indicating that some propagation of figural assignment did occur (because the difference between probe types reflects the propagation of figural assignment from the biased section to the unbiased section); see Figure 6. There were also significant effects of edge grouping $[F(1,15)=24.86, p<.0001]$, region color similarity $[F(1,15)=4.52, p<.049]$, and their interaction $[F(1,15)=7.72, p<.014]$, indicating that these factors had a combined impact on performance. The probe effect interacted with both edge grouping $[F(1,15)=133.46$, $p<.0001]$ and region color similarity $[F(1,15)=10.35$, $p<.006]$, confirming that the difference between figure and ground probes (the critical measure of edge assignment propagation in the present study) depended on edge grouping and was also affected by the presence of region color similarity. As in all of our previous experiments, region color similarity had an effect only when edge grouping was also present, as reflected in the threeway interaction of probe type, edge grouping, and region color similarity $[F(1,15)=12.15, p<.003]$. None of the factors interacted with salience (all $p \mathrm{~s}>$.189), demonstrating that the results were equivalent for the three dotsalience conditions. There was no main effect of salience, either $[F(2,30)=0.37, p<.692]$.

Within each salience condition (assessed by separate $2 \times 2 \times 2$ ANOVAs on the three distinct dot arrangements), there was always an impact of probe type [for salience balanced, $F(1,15)=26.85, p<.0001$; ground salient, $F(1,15)=18.39, p<.001$; figure salient, $F(1,15)=$ $23.18, p<.0001$ ] and of edge grouping [salience-balanced, $F(1,15)=17.77, p<.001$; ground salient, $F(1,15)=$ $22.37, p<.0001$; figure salient, $F(1,15)=10.75, p<$ $.005]$. There was also a three-way interaction for all dot conditions [i.e., between probe type, edge grouping, and region color similarity: for salience-balanced, $F(1,15)=$ $7.03, p<.018$; ground salient, $F(1,15)=5.76, p<.03$; figure salient, $F(1,15)=8.16, p<.01]$. In all cases, this interaction arose because region color similarity mattered for the impact of probe type only when edges were grouped (as in all of our previous experiments). As in Experiment 3, at the request of a reviewer, we tested for effects of the counterbalanced expansion/contraction cue. As in Experiment 3, we found no significant impact on the STEM task: mean RTs of $1,070.6$ msec versus $1,067.2 \mathrm{msec}$ for congruent and incongruent probes, respectively $[t(15)=0.148, p=.881] ; 7.5 \%$ and $7.3 \%$ error rates, respectively $[t(15)=0.718, p=.483]$. These results are consistent with those found in Experiment 3.

The three replications (see Figure 6) of the STEM task in Experiment 4 all converge perfectly with the previous STEM task results from Experiment 3 (compare Figures 5 and 6), as well as with the subjective report results of Experiments 1 and 2, with respect to our factors of main interest. In Experiment 4, further evidence is provided (in addition to that in Experiment 2) that none of our critical effects depends on dot salience's favoring one or other side of the biased section.

\section{EXPERIMENT 5 Extension to Static Displays and the Role of the Occluder}

We next tested whether our new principle - that grouping constrains the propagation of figural edge assignment between edges - would generalize to different displays and grouping factors. We now manipulated edge grouping in a completely different way than via common fate, and we also used a very different local edge-assignment cue for the biased section, now within static displays. In Experiment 5, we returned to phenomenal measures (as in Experiments 1 and 2). We manipulated T-junctions as the local cue for edge assignment within the biased section (see Figures 7A-7D, noting the different vertical extents of the black and white regions at the bottom of each display and the resulting T-junction, as arrowed in Figure 7A). T-junctions have previously been recognized as an important cue to relative depth at edges (Clowes, 1971; Guzman, 1969; Huffman, 1971) and have been used to manipulate edge assignment in some neurophysiological work (see, e.g., Zhou, Friedman, \& von der Heydt, 2000). Furthermore, several computational models of edge assignment (as were mentioned in the introduction) that consider the propagation of such an assignment along complete or partially complete contours actually rely on T-junction depth cues (e.g., Craft et al., 2007; Heitger et al., 1998; Peterhans \& Heitger, 2001; Thielscher \& Neumann, 2008).

In Experiment 5, we also took a closer look at any role for contrast polarity across the critical dividing edges as a grouping factor (previously we had varied contrast polarity only when region color was dissimilar rather than varying this in a fully orthogonal manner; see Experiment 1). Contrast polarity now served as a strictly orthogonal edgegrouping cue (see below). Manipulating just contrast polarity allowed us to hold collinearity constant now between the biased and unbiased dividing edges, while also eliminating common fate, unlike in our four preceding experiments. We now used static rather than moving displays, seeking to generalize our findings.

Finally, in order to understand the role of the occluder that had previously intervened between biased and unbiased dividing edges, and to study how this might affect propagation of edge assignment, we now varied the perceived depth of the potentially occluding horizontal segment that had separated biased and unbiased sections hitherto (see Figure 1A). When this segment appeared to be an occluder, apparently in front of the biased and 


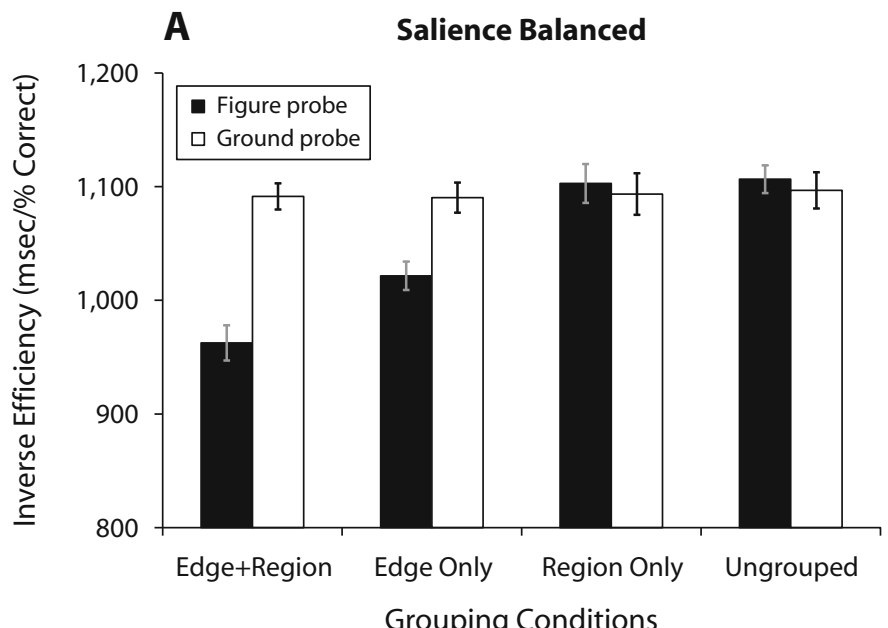

B

Ground Salient

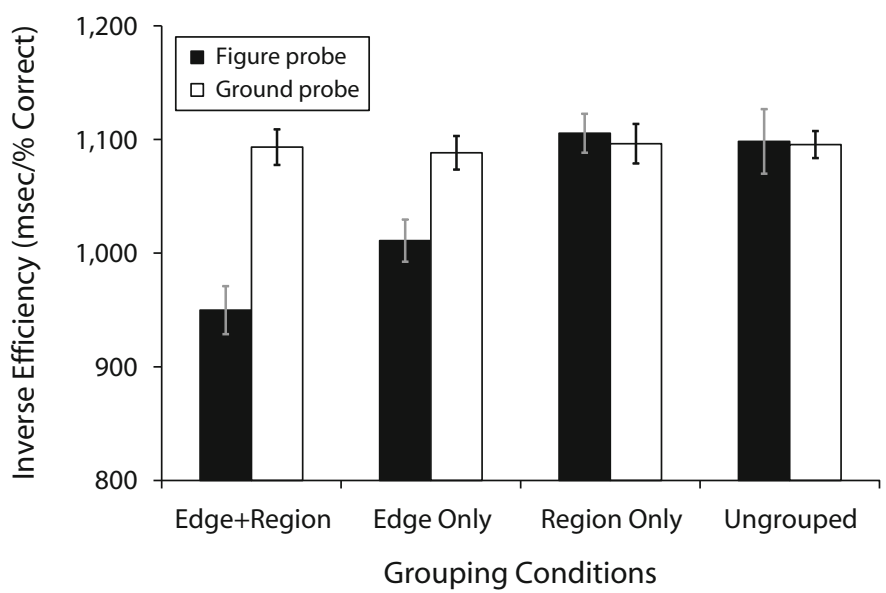

C

Figure Salient

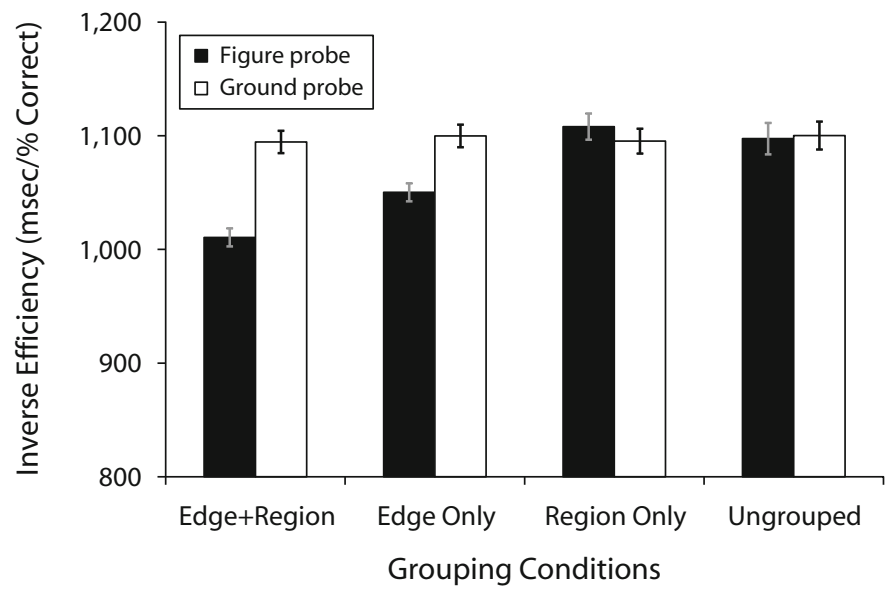

Figure 6. Results of Experiment 4. (A) Results for the saliencebalanced condition (i.e., moving dots on both sides of the biased section in prime). (B) Results for the ground-salient condition (i.e., dots only on the ground side for the biased section in the prime). (C) Results for the figure-salient condition (i.e., dots only on figural side for biased section in prime). Error bars represent $95 \%$ within-subjects confidence intervals. Note the similar outcomes for all three graphs, all of which replicate the pattern from Experiment 3 that is shown in Figure 5. 

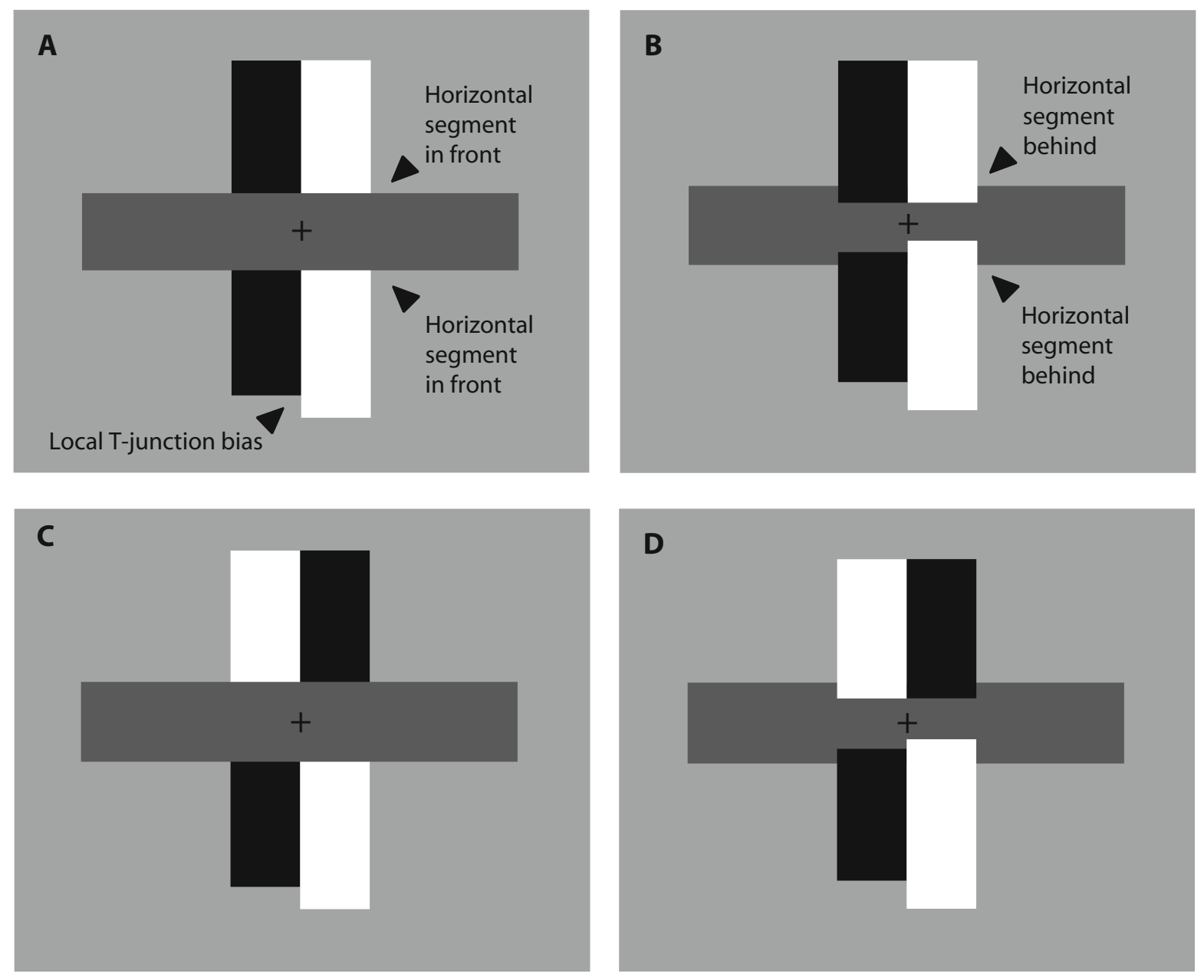

E

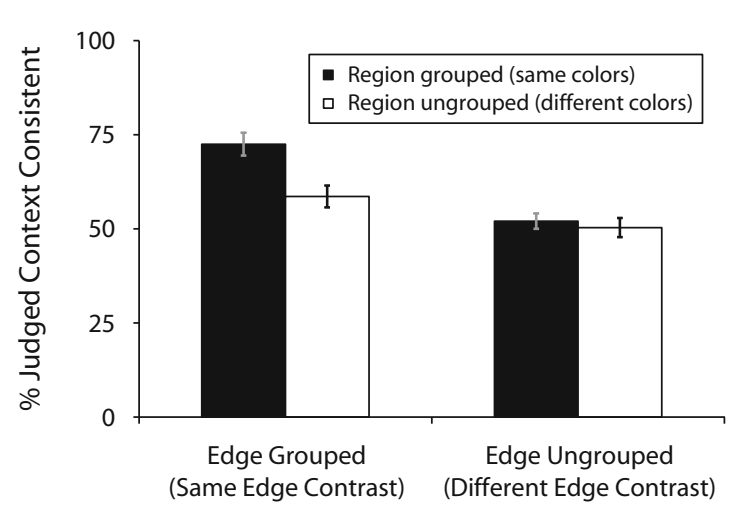

Edge Grouping by Contrast Similarity

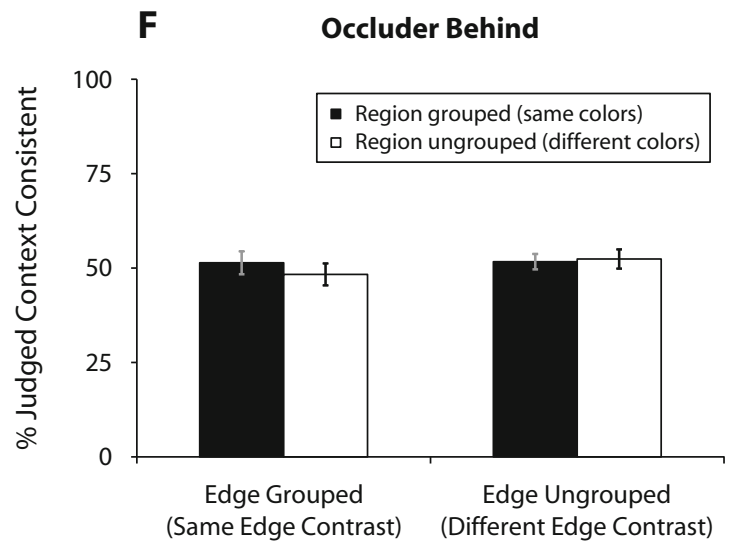

Region Color Similarity

Figure 7. Experiment 5, example displays and results. (A) Horizontal segment serving as an occluder that appears pictorially "in front," with an edge grouping $\times$ edge contrast similarity between biased (bottom) and unbiased (top) sections. Local T-junction cues (see arrow, not present in actual display) assign the dividing edge to the right within the biased section at bottom. (B) The horizontal segment now appears pictorially "behind," so it cannot serve as an occluder, despite edge-contrast similarity between biased (bottom) and unbiased (top) sections. Note that the new pictorial cues to the horizontal segment's being behind actually bring the unbiased and biased sections slightly closer together, but, nevertheless, less propagation of edge assignment is found between them. (C) The horizontal segment pictorially in front (as for panel A), but now with edges in biased and unbiased sections having opposite contrast polarity. (D) The horizontal segment pictorially behind (see also panel B), now with edges in biased and unbiased sections having opposite contrast polarity. (E) Results of Experiment 5 for the conditions with the horizontal segment in front. (F) Results of Experiment 5 for the conditions with the horizontal segment behind, so that it is unlikely to look like an occluder, which eliminates the impact of edge grouping and its interaction with region color similarity. 
unbiased sections (see Figures 7A and 7C), the dividing edges in those two sections could actually be continuous in the external world, being interrupted in the image only by partial occlusion. But when the same horizontal segment clearly appeared pictorially as behind (Figures 7B and 7D) the biased and unbiased sections, as indicated by overlaps that broke the T-junctions where the horizontal section met those sections, this interpretation becomes invalid, with the upper and lower sections now less grouped in that sense. Hence, figural edge assignment may then no longer propagate between the biased and unbiased sections as effectively in cases such Figures 7B and 7D as it does for apparent-occlusion cases, such as Figures 7A and 7C.

\section{Method}

Participants. Twelve people from the local community participated ( 6 male, 6 female; average age $=25.3$ years). All of the participants reported normal vision and gave informed consent. None had taken part in the previous experiments.

Displays and Design. The displays were conceptually similar those in to Experiment 1, but now they did not contain any motion or dots. Instead, the assignment of the biased section's dividing edge was determined locally by T-junctions (see Figures 7A-7D) at the far end of the biased section that was farthest away from the unbiased section (and the horizontal potentially occluding segment; see bottom of each panel in Figures 7A-7D). Region color similarity was analogous to that in Experiment 1, in the sense that either the biased and unbiased sections were composed of both black and white, or one section was colored, whereas the other was black and white; see below. The similarity of edge contrast polarity was now for the first time a fully orthogonal factor, regardless of whether region color was similar or dissimilar between biased and unbiased sections. A final factor was whether the segment intervening between these sections appeared to be in front of (see, e.g., Figures 7A and 7C) or behind (Figures 7B and 7D) both the biased and unbiased sections, as manipulated via pictorial overlap cues.

In the condition with the intervening segment now pictorially behind, we extended the ends of both the biased and unbiased sections into the horizontal intervening segment. This created T-junctions along the outer vertical edges of the black and white regions (i.e., the biased and unbiased section), which caused the horizontal segment to appear behind them (see Figures 7B and 7D). Within the unbiased section, both of the regions protruded $0.5^{\circ}$ into the horizontal segment (upper sections in Figures 7B and 7D). However, in the biased section, the ground region extended $0.5^{\circ}$ into the horizontal segment, whereas the figural region extended $0.8^{\circ}$ into the horizontal segment (lower section in Figures 7B and 7D). This was done for two reasons. First, it maintained the local edge-assignment cue within the biased section that was also present at the other end of the biased section. Second, the extended figural region was designed to eliminate the otherwise possible perception of the biased and unbiased section regions as connecting via an apparent "slit" in the gray section. Only a slit that was cut just so and then perfectly aligned with the edge between the black and white regions would accord with this perception. This perceptual interpretation should thus be highly unlikely, given the visual system's known bias against highly "accidental" interpretations of a scene (Albert \& Hoffman, 1995; Lowe, 1985; Rock, 1983). Accordingly, we expected that the new stimulus configuration would lead to the horizontal segment's being perceived as behind the abutting biased and unbiased sections, hence our description of this as the "behind" condition, even though only pictorial depth cues were utilized.

Procedure. The procedure was the same as that for Experiment 1 , except displays were shown for only $500 \mathrm{msec}$ (intended to minimize any perceptual reversals), unlike the moving displays in Experiment 1 that required longer display times. Trials with $>1^{\circ}$ de- viations from fixation or with pursuit eye movements $\left(>0.5^{\circ}\right.$ regular oscillatory structure) during the displays were excluded (intersubject mean of $0.5 \%$ of trials discarded).

\section{Results and Discussion}

For the biased section, T-junction cues determined phenomenal figural edge assignment as expected in $90.9 \%$ of trials [significantly more than $50 \%$ chance, $t(11)=$ 28.04, $p<.001]$. This did not differ between the conditions when the horizontal segment was in front $(90.0 \%)$ versus when it was behind (91.8\%) $[t(11)=1.63, p<$ .134], confirming that the local bias that we had applied to the biased section via pictorial cues was equally effective in either case. For the unbiased section, the percentage of context-consistent judgments (see Figures 7E and 7F) depended on our new grouping cue of whether the horizontal segment appeared as an occluder $[F(1,11)=14.05, p<$ .003 ], and it also depended on both region color similarity $[F(1,11)=6.25, p<.029]$ and edge-contrast-polarity matching or otherwise $[F(1,11)=19.33, p<.001]$. Occlusion interacted with the region-color-similarity factor $[F(1,11)=5.71, p<.036]$ and the edge-contrast-polarity factor $[F(1,11)=19.61, p<.001]$, because in the absence of apparent occlusion, the other factors had no impact (i.e., all of the segment-behind conditions had chance levels of context-consistent responses when reporting the unbiased section's figural edge assignment; see Figure 7F). By contrast, when the horizontal intervening segment appeared to be an occluder, figural assignment of the dividing edge propagated from the biased to the unbiased section. This propagation was strongest when both region color was similar and contrast polarity of the edges matched; see Figure 7E. These results are logically analogous to those in Experiment 1 (and in all of our other experiments). But they now show that our new principle, whereby grouping constrains propagation of figural edge assignment, extends to very different displays and to different grouping cues.

In particular, the results of Experiment 5 show that our findings on propagation of figural edge assignment from the biased to the unbiased section can generalize to static displays, in which T-junctions serve as the local figural cues (at the extremity of the biased section). The results further show that this propagation is constrained by occlusion relations and by region color similarity and edge contrast polarity, provided that the intervening segment appears to be an occluder, consistent with biased and unbiased edges's potentially reflecting a common edge that is partially occluded.

\section{GENERAL DISCUSSION}

Our results show that the figural assignment of a dividing edge can be affected by contextual information well beyond the two local regions immediately on either side of that edge, providing the basis for a new class of contextual figure-ground cues. But, importantly, not all contextual edges have equal influence. Edge assignment along one dividing edge (in the locally biased section 
for the present study) propagated only to the other dividing edge (in the unbiased section) when the two edges grouped with one another. This gating of edge assignment propagation by grouping was shown for common fate motion and collinearity (Experiments 1-4), and separately for grouping by edge contrast polarity and occlusion (Experiment 5). When the occlusion relation was reversed pictorially, rendering the separate edges perceptually discontinuous (Figures 7B and 7D), propagation of edge assignment from the biased to the unbiased section was eliminated. Region color similarity also had some facilitatory influence on propagation of edge assignment, but could be vetoed by edge ungrouping. In other words, region color similarity mattered only when the dividing edges were grouped, as we confirmed in all five experiments.

It may be worth noting that in the present, we always manipulated region color similarity in the presence of relatively powerful edge-grouping factors (such as contrast polarity and common fate). In the present studies, we did not explore the influence of region color similarity on its own. Moreover, in all of the cases in which edge grouping was absent in our study, one could argue that, in fact, there was a strong edge-ungrouping cue (e.g., different speeds and out-of-phase motion for the common fate manipulations, or opposite contrast polarity in our static experiment). In documenting for the first time the contextual propagation of figure-ground assignment between disconnected elements of a display under the constraint of grouping, our present study raises many new questions for future research. These include whether region color similarity can be effective on its own when edge grouping is kept neutral; what happens if color similarity for potential grounds is manipulated orthogonally to that for potential figures; whether absolute or relative color or contrast values are more critical; and how figural assignment of the unbiased section arises in time, relative to that for the biased section. Future variations on the approach introduced in the present article could address these issues, together with many further interesting questions that arise from our initial demonstrations.

We propose that our existing demonstrations reflect a mechanistic link between perceptual grouping mechanisms and figural edge-assignment mechanisms. From this perspective, propagation of figural edge assignment proceeds when the visual system judges that the edges in question are likely to be related and probably reflect a common external source, as when the edges are grouped together. However, as we briefly discussed when introducing Experiments 2 and 4, it might be possible for one to suggest that attention could play some mediating role, in principle. For instance, we considered (but rejected) the possibility that attention capture by salient dots on the figural side of the biased edge might provide an advantage to that side, with this advantage then potentially propagating via object-based or grouping-constrained attention (see, e.g., Behrmann, Zemel, \& Mozer, 2000; Egly et al., 1994; Matsukura \& Vecera, 2006) to the corresponding side of the unbiased edge. In Experiments 2 and 4, we addressed this issue for the specific proposal that attention might be captured by salient moving dots, by manipulating which side(s) of the biased dividing edge contained such dots. We found that the critical results remained, even when moving dots appeared only on the ground side of the biased dividing edge, thus arguing against any simple account for our results in terms of attention capture by moving dots per se.

It might nevertheless be possible still to argue that, despite this specific result, figural regions tend intrinsically to attract attention following edge assignment to that side (see, e.g., Nelson \& Palmer, 2007, for evidence that this happens for familiar shapes). In such an argument, the figural side of the biased section might thereby become the attended side also, making it potentially ambiguous whether figural assignment per se, or attention (or both) is what propagates to the corresponding side of the unbiased section. We think that our dot-salience manipulation provides some initial evidence against such a proposal, or at least evidence that makes such a proposal less parsimonious. On our account, figural assignment can propagate, under the constraint of grouping, from a biased dividing edge to an unbiased dividing edge. It seems to us that any alternative account, in terms of an intervening role for attention, probably has to postulate a similar causal chain, but with the added premise that redirection of attention might intervene between an initial figural assignment of the biased dividing edge and propagation of the resulting advantage for one side to the corresponding side of the unbiased section, again under the constraint of grouping. Occam's razor would thus suggest that any such intervening role for attention has to be proven rather than merely assumed. Moreover, it is no longer uncontested that figural regions and attended regions are inevitably associated (Huang \& Pashler, 2009). For these reasons, we prefer for now to retain our hypothesis that figural assignment for one edge can propagate to related but separate edges, under the constrain of grouping, without necessarily advocating any essential or additional role for attention. Nevertheless, now that we have established the new principle of figural assignment's propagating under the constraint of perceptual grouping, it may be interesting to study whether this applies equally for attended and unattended stimuli in future variants of the paradigms we have introduced.

Our manipulation of the occluder in Experiment 5 provides a further demonstration, now in relation to pictorial cues for 3-D depth, that when perceptual evidence suggests that the edges and regions in the biased and unbiased sections of the display are unlikely to be related, then figural edge-assignment will not propagate between them. One way of understanding this occluder manipulation is as another example of a grouping manipulation. When the occluder is in front, it suggests that the edges and regions should be completed behind it by virtue of the aligned T-junctions on either side of it, which provide a strong perceptual cue for a partially occluded relation. However, when the occluder is pictorially behind, the evidence that these junctions are actually corners suggests that the two 
sections should not group with one another and thus that the regions should not be interpolated.

Our results provide a new rationale for investigating how neural populations that are involved in visual contour grouping (see, e.g., Polat, Mizobe, Pettet, Kasamatsu, \& Norcia, 1998) may relate to those involved in figural edge assignment (e.g., Zhou et al., 2000). Such interactions have previously been suggested by computational modeling work (Craft et al., 2007; Heitger et al., 1998; Peterhans \& Heitger, 2001; Thielscher \& Neumann, 2008) in which edge assignment caused by T-junctions is considered to propagate along contours. One possible neural correlate for our new results on human perception might be that the neural populations involved in contour grouping (e.g., Polat et al., 1998) are also involved in figural edge assignment (e.g., von der Heydt, Zhou, \& Friedman, 2000; Zhou et al., 2000). Alternatively, two distinct populations (one responsible for grouping, another for edge assignment) might interact. Neural measures in paradigms relating to those that we have introduced in the present article may be required to resolve this.

Our results cast some doubt on Palmer and Rock's (1994b) proposal that figure-ground assignment should always precede grouping. Their claim was largely based on arguments from first principles within their overarching perspective on perceptual organization. They initially proposed that grouping may occur only after figureground organization, arguing that it provides the elements that can then be subject to grouping processes. In the present study, we have clearly established the converse influence for human perception-namely that grouping can affect figure-ground organization. Grouping clearly determined figure-ground assignment for the unbiased section of our displays. But one might still ask whether figural assignment within the biased section preceded, followed, or overlapped with the grouping between biased and unbiased edges and regions. Some of the neural measures, as suggested above, might shed further light on the exact time course of the underlying processes.

Despite the new issues arising for future research, the present results clearly establish a new contextual influence on figure-ground assignment and show that this contextual influence is gated by perceptual grouping. By integrating information about edge assignment only between grouped contextual edges, figure-ground processes may be able to maintain consistent figure-ground organization along edges in which inconsistent local cues or partial occlusion in the image might otherwise disrupt this continuity.

\section{AUTHOR NOTE}

J.L.B. was supported by a Royal Society Postdoctoral Fellowship. J.D. was supported by the Wellcome Trust, MRC, BBSRC, and a Royal Society Anniversary Research Professorship. We thank Sergei Gepshtein for early discussions leading to this work. J.D. is affiliated with both the UCL Institute of Cognitive Neuroscience and the Wellcome Centre for Neuroimaging at UCL. Address correspondence to J. L. Brooks, UCL Institute of Cognitive Neuroscience, University College London, 17 Queen Square, London WC1N 3AR United Kingdom (e-mail: joseph brooks@ucl.ac.uk).

\section{REFERENCES}

Albert, M. K., \& Hoffman, D. D. (1995). Genericity in spatial vision. In R. D. Luce, M. D'Zmura, D. D. Hoffman, G. J. Iverson, \& A. K Romney (Eds.), Geometric representations of perceptual phenomena: Papers in honor of Tarow Indow on his 70th birthday (pp. 95-112). Mahwah, NJ: Erlbaum.

Appelbaum, L. G., Wade, A. R., Vildavski, V. Y., Pettet, M. W., \& Norcia, A. M. (2006). Cue-invariant networks for figure and background processing in human visual cortex. Journal of Neuroscience, 26, 11695-11708.

BARENHOLTZ, E., \& TARR, M. J. (2009). Figure-ground assignment to a translating contour: A preference for advancing vs. receding motion. Journal of Vision, 9(5, Art. 27), 1-9.

BaYlis, G. C., \& CALE, E. M. (2001). The figure has a shape, but the ground does not: Evidence from a priming paradigm. Journal of Experimental Psychology: Human Perception \& Performance, 27, 633-643.

BaYlis, G. C., \& Driver, J. (1995). One-sided edge assignment in vision: 1. Figure-ground segmentation and attention to objects. Current Directions in Psychological Science, 4, 140-146.

BAYLIS, G. C., \& DrIVER, J. (2001). Shape-coding in IT cells generalizes over contrast and mirror reversal, but not figure-ground reversal. Nature Neuroscience, 4, 937-942.

Behrmann, M., Zemel, R. S., \& Mozer, M. C. (2000). Occlusion, symmetry, and object-based attention: Reply to Saiki (2000). Journal of Experimental Psychology: Human Perception \& Performance, 26, 1497-1505.

Clowes, M. B. (1971). On seeing things. Artificial Intelligence, 2, 79116.

Craft, E., Schutze, H., Niebur, E., \& von der Heydt, R. (2007). A neural model of figure-ground organization. Journal of Neurophysiology, 97, 4310-4326.

Driver, J., \& BAYLIS, G. C. (1996). Edge-assignment and figure-ground segmentation in short-term visual matching. Cognitive Psychology, 31, 248-306.

EgLy, R., Driver, J., \& RAFAL, R. D. (1994). Shifting visual attention between objects and locations: Evidence from normal and parietal lesion subjects. Journal of Experimental Psychology: General, 123, $161-177$

Franconeri, S. L., \& Simons, D. J. (2005). The dynamic events that capture visual attention: A reply to Abrams and Christ (2005). Perception \& Psychophysics, 67, 962-966.

Guzman, A. (1969). Decomposition of a visual scene into threedimensional bodies. In A. Griselli (Ed.), Automatic interpretation and classification of images (pp. 243-276). New York: Academic Press.

Heitger, F., von der Heydt, R., Peterhans, E., Rosenthaler, L., \& Kubler, O. (1998). Simulation of neural contour mechanisms: Representing anomalous contours. Image \& Vision Computing, 16, 407-421.

Hillstrom, A. P., \& YanTIS, S. (1994). Visual motion and attentional capture. Perception \& Psychophysics, 55, 399-411.

HuAng, L., \& Pashler, H. (2009). Reversing the attention effect in figure-ground perception. Psychological Science, 20, 1199-1201.

HufFMan, D. A. (1971). Impossible objects as nonsense sentences. In B. Meltzer \& D. Michie (Eds.), Machine intelligency (Vol. 6, pp. 295323). Edinburgh: Edinburgh University Press.

Hulleman, J., \& Humphreys, G. W. (2004). A new cue to figureground coding: Top-bottom polarity. Vision Research, 44, 27792791.

Kanizsa, G., \& Gerbino, W. (1976). Convexity and symmetry in figure-ground organization. In M. Henle (Ed.), Vision and artifact (pp. 25-32). New York: Springer.

KOURTZI, Z., \& KANWISHER, N. (2001). Representation of perceived object shape by the human lateral occipital complex. Science, 293, 1506-1509.

Lowe, D. G. (1985). Perceptual organization and visual recognition. Boston: Kluwer

Matsukura, M., \& Vecera, S. P. (2006). The return of object-based attention: Selection of multiple-region objects. Perception \& Psychophysics, 68, 1163-1175. 
Metzger, W. (1953). Gesetze des Sehens. Frankfurt-am-Main: Waldemar Kramer.

Nelson, R., \& Palmer, S. E. (2007). Familiar shapes attract attention in figure-ground displays. Perception \& Psychophysics, 69, 382-392.

PaLmer, S. E. (1999). Vision science: Photons to phenomenology. Cambridge, MA: MIT Press.

PALmer, S. E., \& Brooks, J. L. (2008). Edge-region grouping in figureground organization and depth perception. Journal of Experimental Psychology: Human Perception \& Performance, 34, 1353-1371.

Palmer, S., \& Rock, I. (1994a). On the nature and order of organizational processing: A reply to Peterson. Psychonomic Bulletin \& Review, 1, 515-519.

Palmer, S., \& Rock, I. (1994b). Rethinking perceptual organization: The role of uniform connectedness. Psychonomic Bulletin \& Review, 1, 29-55.

Peterhans, E., \& Heitger, F. (2001). Simulation of neuronal responses defining depth order and contrast polarity at illusory contours in monkey area V2. Journal of Computational Neuroscience, 10, 195-211.

Peterson, M. A. (1994). Object recognition processes can and do operate before figure-ground organization. Current Directions in Psychological Science, 3, 105-111.

Peterson, M. A., \& Gibson, B. S. (1994a). Must figure-ground organization precede object recognition? An assumption in peril. Psychological Science, 5, 253-259.

Peterson, M. A., \& Gibson, B. S. (1994b). Object recognition contributions to figure-ground organization: Operations on outlines and subjective contours. Perception \& Psychophysics, 56, 551-564.

Peterson, M. A., \& Salvagio, E. (2008). Inhibitory competition in figure-ground perception: Context and convexity. Journal of Vision, 8(16, Art. 4), 1-13.

Polat, U., Mizobe, K., Pettet, M. W., Kasamatsu, T., \& Norcia, A. M. (1998). Collinear stimuli regulate visual responses depending on cell's contrast threshold. Nature, 391, 580-584.

Rock, I. (1983). The logic of perception. Cambridge, MA: MIT Press.

Rubin, E. (1921). Visuell Wahrgenommene Figuren. Copenhagen: Glydendalske.
Stevens, K. A., \& Brookes, A. (1988). The concave cusp as a determiner of figure-ground. Perception, 17, 35-42.

Thielscher, A., \& Neumann, H. (2008). Globally consistent depth sorting of overlapping 2D surfaces in a model using local recurrent interactions. Biological Cybernetics, 98, 305-337.

Townsend, J. T., \& Ashby, F. G. (1978). Methods of modeling capacity in simple processing systems. In N. J.Castellan \& F. Restle (Eds.), Cognitive theory (pp. 199-239). Hillsdale, NJ: Erlbaum.

Vecera, S. P., Flevaris, A. V., \& Filapek, J. C. (2004). Exogenous spatial attention influences figure-ground assignment. Psychological Science, 15, 20-26.

Vecera, S. P., Vogel, E. K., \& Woodman, G. F. (2002). Lower region: A new cue for figure-ground assignment. Journal of Experimental Psychology: General, 131, 194-205.

von Der Heydt, R., Zhou, H., \& Friedman, H. S. (2000). Representation of stereoscopic edges in monkey visual cortex. Vision Research, 40, 1955-1967.

WAGner, G., \& Boynton, R. M. (1972). Comparison of four methods of heterochromatic photometry. Journal of the Optical Society of America, 62, 1508-1515.

Yonas, A., Craton, L. G., \& Thompson, W. B. (1987). Relative motion: Kinetic information for the order of depth at an edge. Perception \& Psychophysics, 41, 53-59.

Zhou, H., Friedman, H. S., \& von der Heydt, R. (2000). Coding of border ownership in monkey visual cortex. Journal of Neuroscience, 20, 6594-6611.

\section{SUPPLEMENTAL MATERIALS}

Examples of the animations discussed in this article and error and RT results for Experiments 3 and 4 may be downloaded from http://app .psychonomic-journals.org/content/supplemental.

(Manuscript received October 19, 2009; revision accepted for publication January 31, 2010.) 